\title{
Filament-assisted mode conversion in magnetized plasmas
}

\author{
W. Tierens, ${ }^{1, \text { a) }}$ W. Zhang, ${ }^{1, \text { b) }}$ J.R. Myra, ${ }^{2}$ and EUROfusion MST1 Team $^{3}$ \\ ${ }^{1)}$ Max Planck Institute of Plasma Physics, D-85748 Garching, Germany \\ 2) Lodestar Research Corporation, CO 80301 Boulder, Colorado, USA \\ ${ }^{3)}$ See B. Labit et al 2019 Nucl. Fusion 59086020
}

(Dated: 11 December 2019)

At density filaments in magnetized plasmas, electromagnetic waves are guided along the filament ("wave-filament bound states"). Several wave-filament bound states exist beyond those predicted in ${ }^{1}$. The new bound states occur under experimentally relevant conditions, and are especially common under typical NSTX conditions, where they are a plausible mechanism for the experimentally observed power losses in the edge plasma.

In experimental magnetic confinement fusion devices, density filaments occur $^{1-6}$, with which electromagnetic waves intended to heat the plasma interact. Such interactions are usually studied from the point of view of wave scattering ${ }^{1,7-9}$ : one wishes to ensure that not too much useful power will be scattered in unforeseen directions by the turbulent edge plasma. The possibility of mode conversion at density filaments in the edge plasma was briefly raised in $^{1}$, and is the central topic of this paper.

We consider waves in the Ion Cyclotron Range of Frequencies (ICRF), where the antenna launches the "Fast Wave" (FW), which will mode-convert to the much shorterwavelength "Slow Wave" (SW). Figure 1 shows typical wavelengths for these modes ${ }^{10}$.

To investigate this mode conversion, we will make use of exact solutions of Maxwell's equations for the scattering of an incident planar Fast Wave at infinitely long cylinders. Such solutions take the form of infinite series over eigenmodes in a cylindrical coordinate system, known as a Mie series ${ }^{12}$, after Gustav Mie who first derived them in $1908^{13}$. More re-

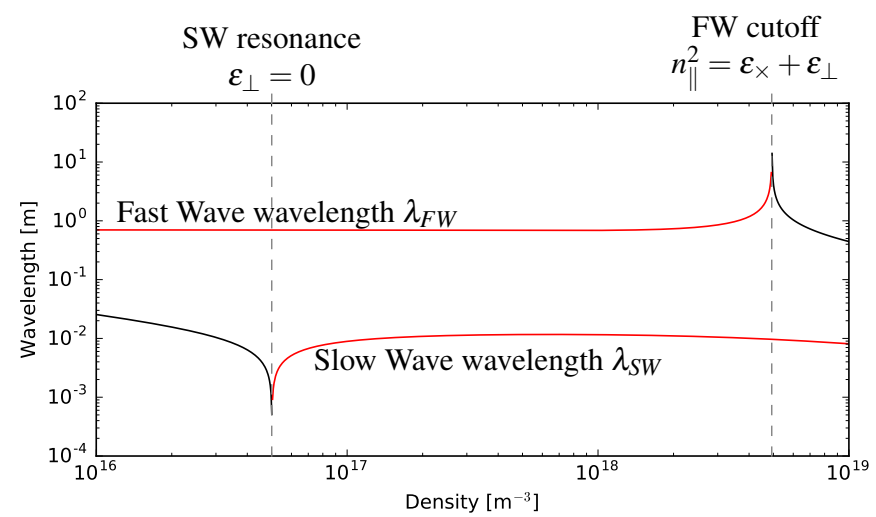

FIG. 1. Perpendicular wavelengths $\frac{2 \pi}{\left|k_{\perp}\right|}$ of the Fast Wave and the Slow Wave in a deuterium plasma at various densities, with $B_{0}=2 \mathrm{~T}$, $f=36.5 \mathrm{MHz}$ (ICRF), $k_{\|}=\frac{n_{\|} \omega}{c}=9 \mathrm{~m}^{-1}$ as is typical in $\mathrm{AUG}^{11}$. Black curves are where the wave is propagating $\left(k_{\perp}\right.$ real $)$, red curves are where it is evanescent $\left(k_{\perp}\right.$ imaginary).

\footnotetext{
a)Electronic mail: wouter.tierens@ipp.mpg.de

b)Electronic mail: wei.zhang@ipp.mpg.de
}

cently, the Mie series was generalized to plane wave scattering at cylindrical density filaments ${ }^{8}$ or spherical density blobs ${ }^{7}$ in magnetized plasma.

The Mie solution is an exact solution of the wave equation

$$
\nabla \times \nabla \times \vec{E}+\frac{\omega^{2}}{c^{2}} \varepsilon \vec{E}=0
$$

where $\varepsilon$ is a tensor which takes one of two values in a cylindrical $(r, \theta, z)$ coordinate system centered on a cylindrical filament of radius $r_{F}$ :

$$
\varepsilon=\left\{\begin{array}{lll}
\varepsilon_{F} & r<r_{F} & \text { in cylindrical Filament } \\
\varepsilon_{B} & r \geq r_{F} & \text { in Background plasma }
\end{array}\right.
$$

Furthermore, the confining magnetic field is assumed to be aligned with the filament, in the $z$ direction of the coordinate system, so the dielectric tensor has the form

$$
\varepsilon=\left[\begin{array}{ccc}
\varepsilon_{\perp} & -i \varepsilon_{\times} & 0 \\
i \varepsilon_{\times} & \varepsilon_{\perp} & 0 \\
0 & 0 & \varepsilon_{\|}
\end{array}\right]
$$

where $\varepsilon_{\perp}, \varepsilon_{\times}$and $\varepsilon_{\|}$depend on the plasma composition ${ }^{10}$.

This approach allows us to exactly decompose the total electric field as a sum of a contribution due to the Fast Wave and a contribution due to the Slow Wave. We will quantify the degree to which an electric field component is due to the Fast Wave or the Slow Wave as

$$
\beta=\frac{\mid \mathrm{SW} \text { contribution } \mid}{\mid \text { FW contribution }|+| \mathrm{SW} \text { contribution } \mid}
$$

We must impose, on every azimuthal mode $m$ in the cylindrical coordinate system, four boundary conditions at $r=r_{F}$ : the tangential components of both the electric field and the magnetic field should be continuous ${ }^{8}$. Correspondingly, there are four amplitude degrees of freedom: amplitudes for the Fast and Slow wave in the filament, and for the scattered Fast and Slow wave in the background plasma, in addition to the known amplitude of the incident wave. The four continuity conditions are a $4 \times 4$ system of equations

$$
M\left[\begin{array}{l}
\text { Filament FW ampl. } \\
\text { Filament SW ampl. } \\
\text { Scattered FW ampl. } \\
\text { Scattered SW ampl. }
\end{array}\right]=\left[\begin{array}{c}
\text { Incident FW } \\
E_{z}, E_{\theta}, B_{z}, B_{\theta} \\
\text { on interface }
\end{array}\right]
$$


which couples all four degrees of freedom, that is, we should expect the Fast Wave to couple to the Slow Wave at the filament interface.

Suppose the boundary condition matrix $M$ in (5) has zero determinant. Then, there is some nonzero amplitude vector that is a solution of (5) even in the absence of incident fields. This represents a wave-filament bound state, a wave which is localized around the filament and propagates along the filament, perhaps analogous to a surface wave ${ }^{14,15}$ on the filament interface. Near $|M|=0$ the mode-converted Slow Wave can have an arbitrarily large amplitude ("mode conversion resonance"1). In reality, the amplitude is limited by collisionality and by the fast radial movement $\left(10^{2}-10^{3} \mathrm{~m} / \mathrm{s}\right)$ of the filaments: the resonance condition only holds at some specific radial location(s), in which the filament does not spend much time (filament diameter/radial velocity $\approx 10^{3} \mathrm{RF}$ periods). Other factors which might limit the amplitude include the density gradient at the filament interface, which in reality is steep rather than a step function, and the fact that at any given time only one $k_{\|}$mode can be resonant, which limits the fraction of the wave energy that can seep into the resonance.

Figure 2 shows generic examples of mode conversion from the Fast Wave to the Slow Wave, in three qualitatively different cases. In case A, all waves are evanescent both in the background plasma and in the filament. As expected, a ring of evanescent $\mathrm{SW} E_{z}$ amplitude forms at the filament surface. In case $\mathrm{B}$, the only propagating wave mode is the Slow Wave in the filament, which gives rise to a standing wave of $\mathrm{SW} E_{z}$ in the filament. In case $C$, the only propagating wave mode is the Fast Wave in the filament, and again a ring of evanescent SW $E_{z}$ amplitude forms at the filament surface. The generic cases in this figure have a parallel electric field that is dominated by the contribution of the mode-converted Slow Wave, but the parallel electric field strength remains only a small fraction of the unperturbed perpendicular electric field due to the incident fast wave.

Figure 3 shows parameter scans of the "mode conversion efficiency" $\frac{\max \mathrm{SW}\left|E_{z}\right| \text { in filament }}{\text { incident } \mathrm{FW}\left|E_{\perp}\right|}$ over the filament density, the background density, and the filament radius. Other parameters are the same as in figure 1. High mode conversion efficiency, i.e. bright curves in figure 3 , indicates a resonance $|M|=0$, or possibly a pseudo-resonance $|M| \approx 0$. Naturally, no mode conversion occurs when $n_{F}=n_{B}$, which explains the dark diagonals. The SW-filament bound states in the lower right blocks ${ }^{1}$, the new SW-FW-filament bound states in the upper left blocks, and the evanescent resonances will be discussed in the next paragraphs. A resonance condition $|M|=0$ exists for every azimuthal mode number $m$, but those at high $m$ give rise to very 'thin' resonances, which are easily missed by the finite resolution $(500 \times 500)$ in figure 3 . A filament starts on the diagonal, as it moves outward towards the tokamak edge, it moves leftward in figure 3, during which it may spend some time in a near-resonant state, but this can only have much effect if the resonance is 'wide', so it stands to reason that only the 'wide' resonances which are visible in figure 3 will have physical relevance.

The SW-filament bound state ${ }^{1}$ occurs where the density inside the filament is such that the Slow Wave propagates,

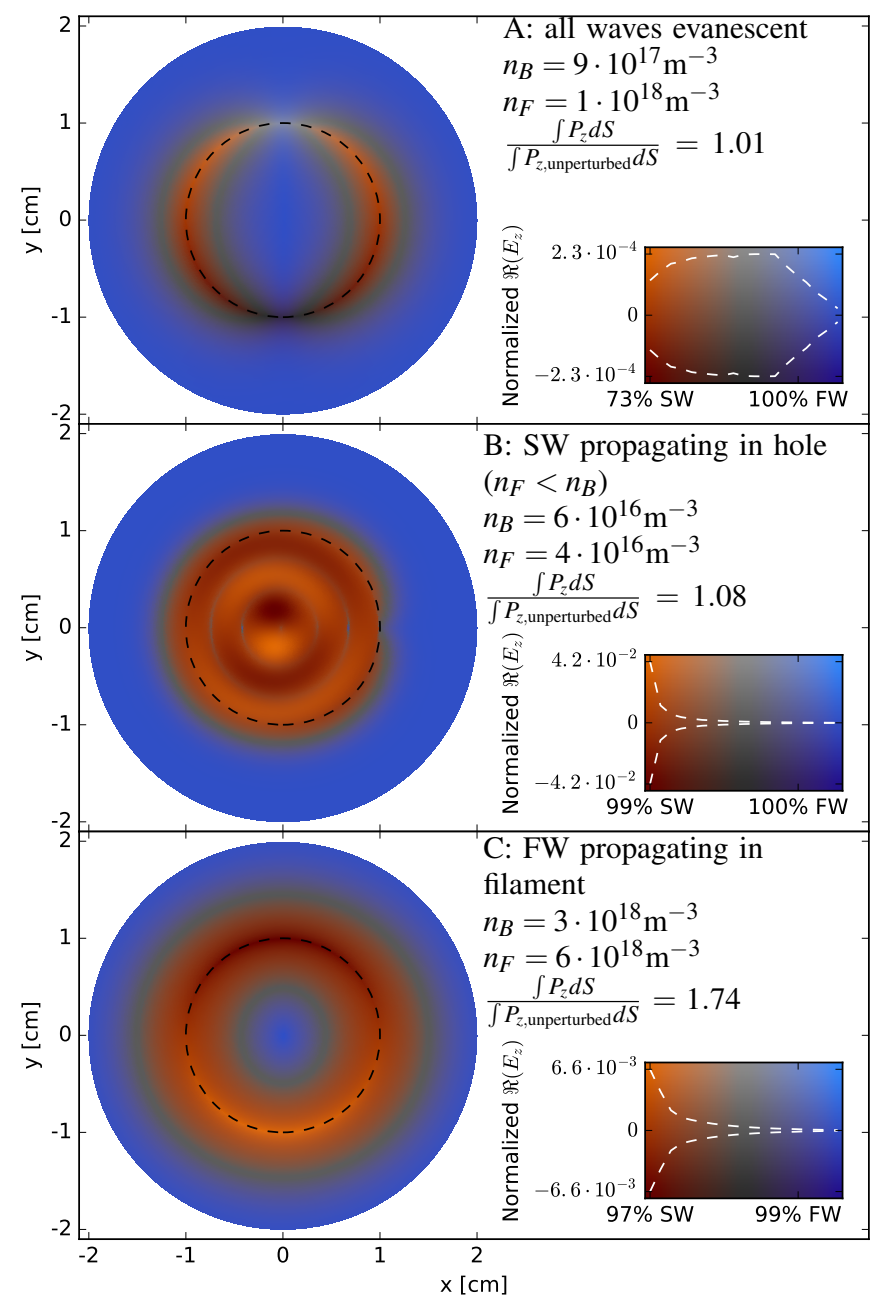

FIG. 2. Mode conversion at a cylindrical density filament. The parallel $z$ component of the electric field is plotted in a cylindrical coordinate system centered on the filament. It is normalized to the unperturbed $\left|E_{\perp}\right|$. The $2 \mathrm{D}$ color scale shows both $\frac{\Re\left(E_{z}\right)}{\text { unperturbed }\left|E_{\perp}\right|}$ using the bright-dark axis, and to what extent $E_{z}$ is due to the Fast Wave or to the Slow Wave using the blue-orange axis (this is $\beta$ defined in eq. (4)). The dashed black circle is the boundary of the density filament with a radius of $r_{F}=1 \mathrm{~cm}$. The filament density $n_{F}$, background density $n_{B}$, and ratio of filament-integrated parallel Poynting fluxes are also shown. Other parameters are as in figure 1.

while it is evanescent in the background. The Slow Wave remains "trapped" as a standing wave in the filament. Myra and D'Ippolito ${ }^{1}$ considered the SW and FW separately, and treated their coupling perturbatively. This allowed them to predict the SW-filament bound state, but not the other bound states that we will descibe later.

As the wavelength of the SW decreases with increasing density before it becomes evanescent, many (in principle infinitely many) resonances exist where the SW-filament bound state is strongly excited. An approximate resonance condition 


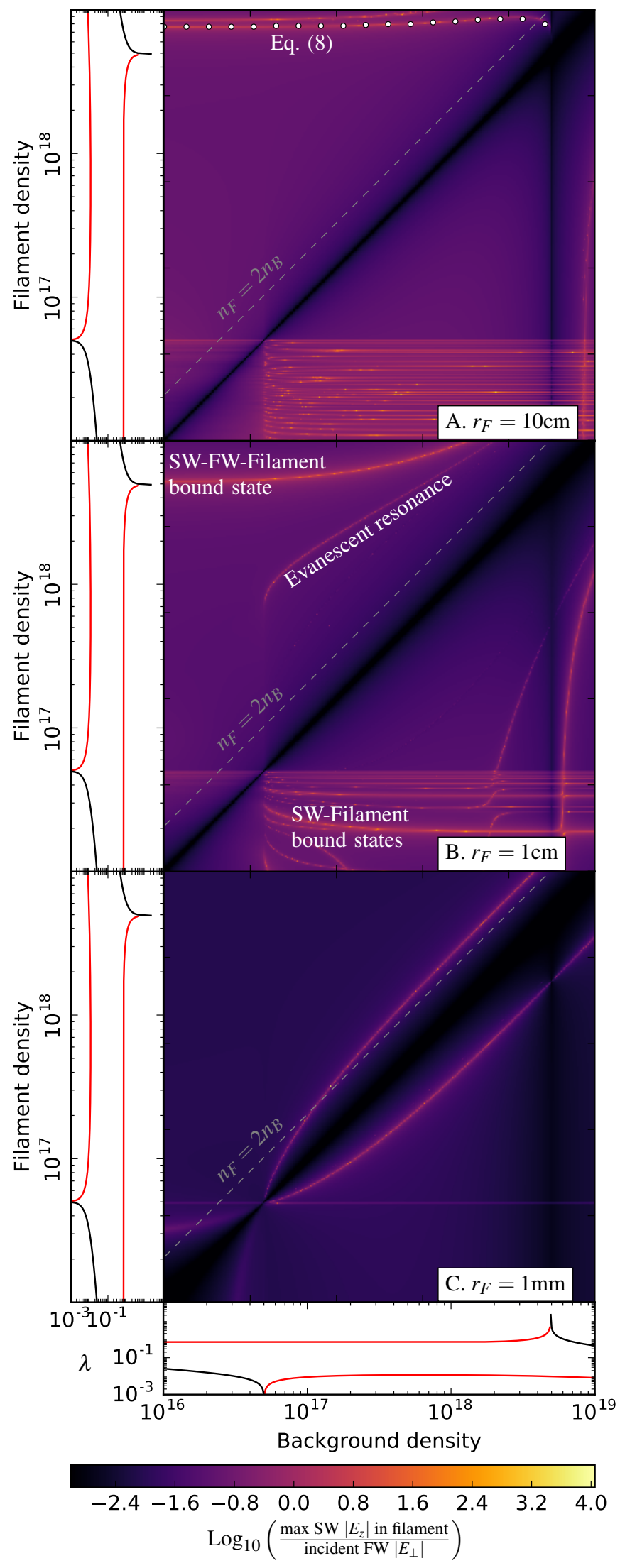

FIG. 3. Mode conversion efficiency vs. filament and background densities. Other parameters are as in figure 1. for these states is ${ }^{1}$

$$
\begin{array}{r}
\frac{\varepsilon_{\perp, B} k_{\perp, B} L_{B} H_{B, m}^{\prime}+\varepsilon_{\times, B} \frac{m}{r_{F}} L_{B} H_{B, m}}{H_{B, m}}= \\
\frac{\varepsilon_{\perp, F} k_{\perp, F} L_{F} J_{F, m}^{\prime}+\varepsilon_{\times, F} \frac{m}{r_{F}} L_{F} J_{F, m}}{J_{F, m}}
\end{array}
$$

where the subscripts $B$ and $F$ indicate Background and Filament quantities. The $k_{\perp}$ are the radial wavenumbers of the Slow Wave. $H$ and $J$ are Hankel and Bessel functions, evaluated at $k_{\perp} r_{F}$. L is defined in the Filament and in the Background as

$$
L_{F}=\frac{-i k_{\|} \lambda_{0}^{2}}{\varepsilon_{\perp, F}-n_{\|}^{2}} \quad L_{B}=\frac{-i k_{\|} \lambda_{0}^{2}}{\varepsilon_{\perp, B}-n_{\|}^{2}}
$$

$\lambda_{0}$ is the vacuum wavelength, $k_{\|}=k_{z}$ the parallel wavenumber, and $m$ is the azimuthal mode number.

The SW-FW-filament bound state is a new type of bound state. It occurs when the Fast Wave is evanescent in the background but propagating in the filament, and the Slow Wave is evanescent in both. This state is qualitatively distinct from the SW-filament bound state in that it is now the Fast Wave which is "trapped inside", and the resonances of this state involve an increase in both the Fast Wave and the Slow Wave amplitude inside the filament.

Recall that the bound states occur where the determinant of the boundary condition matrix is 0 . For large filaments with $r_{F} \gg \frac{2 \pi}{\left|k_{\perp, S W}\right|}$, most determinant terms can be neglected and the $m=1$ resonances are approximately given by

$$
\begin{array}{r}
\frac{J_{0}\left(k_{F} r_{F}\right)}{J_{1}\left(k_{F} r_{F}\right)} k_{B} r_{F}-\frac{H_{0}\left(k_{B} r_{F}\right)}{H_{1}\left(k_{B} r_{F}\right)} k_{F} r_{F} \\
=\left(1-i \frac{E_{F, r}}{E_{F, \theta}}\right) \frac{k_{B}}{k_{F}}-\left(1-i \frac{E_{B, r}}{E_{B, \theta}}\right) \frac{k_{F}}{k_{B}}
\end{array}
$$

where $E_{F}, E_{B}$ are the polarisation of the Fast Wave in the Filament and the Background plasma, and $k_{F}, k_{B}$ are the radial wavenumbers of the Fast Wave in the Filament and the Background plasma. The white dotted curve in figure 3.A is the solution of (8).

In figure 3 , for the smaller filaments $\left(r_{F}=1 \mathrm{~cm}, r_{F}=1 \mathrm{~mm}\right)$, we also see bound states in the region where all waves are evanescent. We call these new bound states "evanescent resonances". These are most likely to occur experimentally, since they require the least extreme density ratio $\frac{n_{F}}{n_{B}}$, among all resonances with $n_{F}>n_{B}$. In experimental fusion devices, this ratio follows a gamma distribution ${ }^{16,17}$ with mean $\frac{n_{F}}{n_{B}} \approx 2$ on ASDEX Upgrade ${ }^{6,18}$, and slightly more on $\operatorname{NSTX}^{19}$, meaning that only a thin strip around the diagonals in figure 3 is typical. Larger values can occur intermittently, but are relatively rare. The required density ratio is closest to 1 for small filaments (fig. 3.C), small $k_{\|}$, or high azimuthal mode numbers.

The evanescent resonance indicated in figure 3 is due to a zero of the boundary condition matrix determinant at $m=$ 2. For even higher azimuthal mode numbers, the boundary condition matrix determinant has roots still closer to $n_{F}=n_{B}$, but those are not efficiently excited by the incident plane wave. 


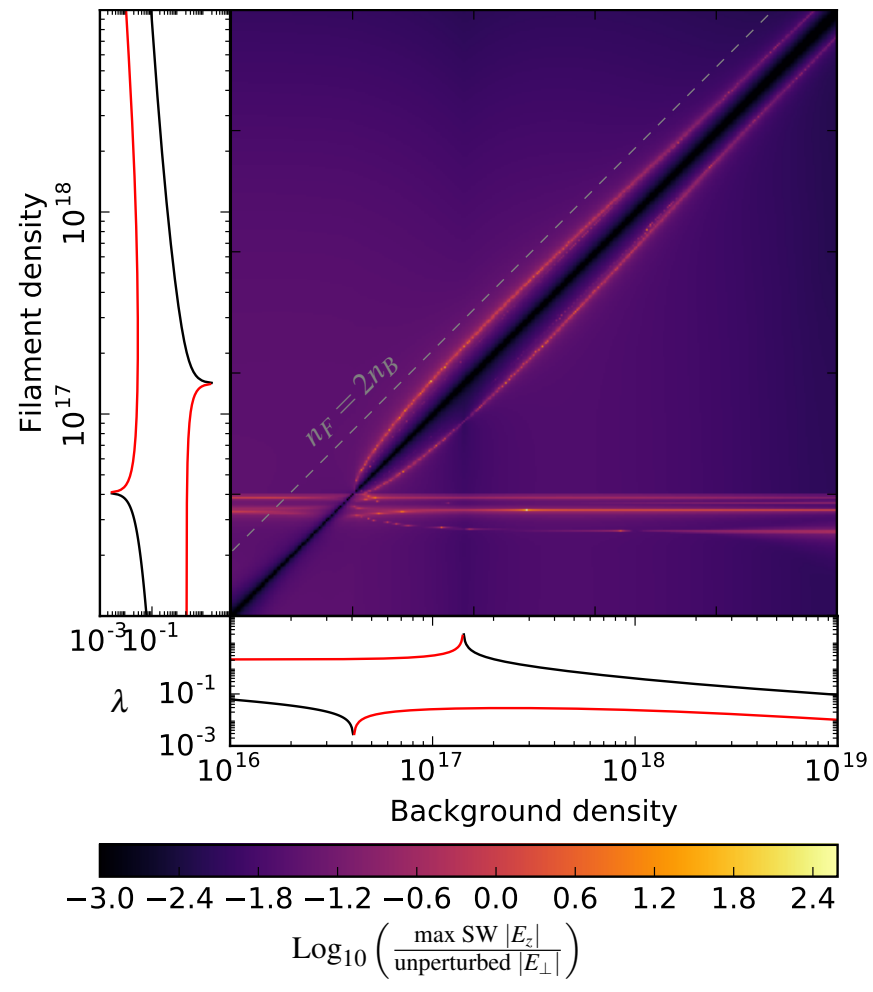

FIG. 4. Mode conversion efficiency for parameters typical in $\mathrm{NSTX}^{20,23-25}$. Edge density $10^{17}-10^{19} \mathrm{~m}^{-3}, B_{0}=0.55 \mathrm{~T}, f=$ $30 \mathrm{MHz}, k_{\|}=3 \mathrm{~m}^{-1}, r_{F}=1 \mathrm{~cm}$. A bound state exists at realistic density ratios. This remains true also for lower magnetic field $\left(B_{0}=\frac{0.55}{2} \mathrm{~T}\right)$ and larger filaments $\left(r_{F}=5 \mathrm{~cm}\right)$ in the NSTX edge plasma.

Figure 4 shows the wave-filament bound states under typical NSTX conditions. They continue to exist in the region where the background FW is propagating, but there they are pseudo-resonances: $|M|$ is small but not exactly 0 . Under these conditions, the wave-filament bound states occur at realistic density ratios $\frac{n_{F}}{n_{B}}<2$ (contrast with figure 3.B, which represents typical ASDEX Upgrade conditions, where the bound states require much higher density ratios). Thus, the wavefilament bound states are predicted to be common in NSTX, and to carry RF power in the parallel direction away from the antenna, which makes them a perfect candidate for the physical mechanism behind the experimentally observed power losses in the NSTX edge plasma. In visible light, filaments in NSTX show up as bright field-aligned spirals. They carry a large fraction of the antenna power along the parallel direction away from the antenna ${ }^{20}$, eventually depositing it on the divertors, where part of it is likely absorbed via sheath rectification $^{21,22}$, a process whose efficiency is enhanced by the increased $E_{\|}$in the wave-filament bound state.

In this paper we discussed mode conversion from the Fast Wave to the Slow Wave at density filaments in magnetized plasma. We have given a non-exhaustive classification of the wave-filament bound states and the mode conversion resonances. In addition to the SW-filament bound state predicted by $^{1}$, we found two new bound states that may have physical relevance: the evanescent resonance and the SW-FW-filament bound state. The evanescent resonance occurs under experimentally relevant circumstances, and is a plausible mechanism for the experimentally observed parallel transport of RF power on NSTX: bound states could be excited by the ICRF waves incident upon the filament in front of the antenna, and then continue propagating along the filament even far away from the antenna, where there are no incident fields. This explanation is consistent with the observed occurence of this parallel transport on NSTX but not on other machines, and is consistent with experimentally observed greater power loss for low $k_{\|}$. It also naturally explains the observed origin of the parallel RF power flow from the SOL in front of the antenna rather than from the antenna surface itself ${ }^{26}$. It does not contradict any of the existing proposed explanations: Wavefilament bound states could act in synergy with the "opening" of the boundary plasma to FW propagation ${ }^{27}$, and with sheath rectification and the subsequent parallel spread of DC bias via DC current transport ${ }^{28-30}$. In future work, we will use Finite Element calculations to ascertain to what extent the claims in this paper depend on the unrealistic assumptions made in the Mie solution, such as non-circular filament cross sections, steep but continuous density gradients at the filament interface, and nonconstant background density.

\section{ACKNOWLEDGMENTS}

We acknowledge input from Peter Manz (Max Planck Institute of Plasma Physics) on realistic values of $\frac{n_{F}}{n_{B}}$, from Lee Berry (Oak Ridge National Laboratory) on the Poynting flux calculation in figure 2 .

This work has been carried out within the framework of the EUROfusion Consortium and has received funding from the Euratom research and training programme 2014-2018 and 2019-2020 under grant agreement No 633053. The views and opinions expressed herein do not necessarily reflect those of the European Commission.

One of us was also supported by a U.S. Department of Energy Scientific Discovery through Advanced Computing Initiative Contract DE-AC05-00OR22725.

${ }^{1}$ J. R. Myra and D. A. D'Ippolito, "Scattering of radio frequency waves by blob-filaments," Physics of Plasmas 17, 102510 (2010).

${ }^{2}$ N. Katz, J. Egedal, W. Fox, A. Le, and M. Porkolab, "Experiments on the propagation of plasma filaments," Physical review letters 101, 015003 (2008).

${ }^{3}$ P. Manz, T. T. Ribeiro, B. D. Scott, G. Birkenmeier, D. Carralero, G. Fuchert, S. H. Müller, H. W. Müller, U. Stroth, and E. Wolfrum, "Origin and turbulence spreading of plasma blobs," Physics of Plasmas 22, 022308 (2015).

${ }^{4}$ G. Fuchert, G. Birkenmeier, D. Carralero, T. Lunt, P. Manz, H. W. Müller, B. Nold, M. Ramisch, V. Rohde, and U. Stroth, "Blob properties in L-and H-mode from gas-puff imaging in ASDEX upgrade," Plasma Physics and Controlled Fusion 56, 125001 (2014).

${ }^{5}$ D. A. D'ippolito, J. R. Myra, and S. J. Zweben, "Convective transport by intermittent blob-filaments: Comparison of theory and experiment," Physics of Plasmas 18, 060501 (2011).

${ }^{6}$ G. Birkenmeier, F. Laggner, M. Willensdorfer, T. Kobayashi, P. Manz, E. Wolfrum, D. Carralero, R. Fischer, B. Sieglin, G. Fuchert, et al., "Magnetic field dependence of the blob dynamics in the edge of ASDEX up- 
grade L-mode plasmas,” Plasma Physics and Controlled Fusion 56, 075019 (2014).

${ }^{7}$ A. Ram, K. Hizanidis, and Y. Kominis, "Scattering of ECRF waves by edge density blobs and fluctuations in tokamak plasmas," in EPJ Web of Conferences, Vol. 32 (EDP Sciences, 2012) p. 01003.

${ }^{8}$ A. Ram and K. Hizanidis, "Scattering of radio frequency waves by cylindrical density filaments in tokamak plasmas," Physics of Plasmas 23, 022504 (2016).

${ }^{9}$ P. T. Bonoli and O. Edward, "Accessibility and energy depositon of lowerhybrid waves in a tokamak with density fluctuations," Physical Review Letters 46, 424 (1981).

${ }^{10}$ T. Stix, Waves in plasmas (Springer Science \& Business Media, 1992).

${ }^{11}$ V. Bobkov, F. Braun, R. Dux, A. Herrmann, H. Faugel, H. Fünfgelder, A. Kallenbach, R. Neu, J.-M. Noterdaeme, R. Ochoukov, et al., "First results with 3-strap ICRF antennas in ASDEX upgrade," Nuclear Fusion 56, 084001 (2016).

${ }^{12}$ J. Li and B. Shanker, "Time-dependent Debye-Mie series solutions for electromagnetic scattering," IEEE Transactions on Antennas and Propagation 63, 3644-3653 (2015).

${ }^{13} \mathrm{G}$. Mie, "Beiträge zur Optik trüber Medien, speziell kolloidaler Metallösungen," Annalen der physik 330, 377-445 (1908).

${ }^{14}$ Y. M. Aliev, Y. M. Aliev, H. Schlüter, H. Schlüter, and A. Shivarova, Guided-wave-produced plasmas, Vol. 24 (Springer Science \& Business Media, 2000).

${ }^{15}$ A. Messiaen and R. Weynants, "ICRH antenna coupling physics and optimum plasma edge density profile. application to ITER," Plasma Physics and Controlled Fusion 53, 085020 (2011).

${ }^{16}$ O. E. Garcia, R. Kube, A. Theodorsen, and H. Pécseli, "Stochastic modelling of intermittent fluctuations in the scrape-off layer: Correlations, distributions, level crossings, and moment estimation," Physics of Plasmas 23, 052308 (2016).

${ }^{17}$ O. E. Garcia, R. Kube, A. Theodorsen, B. LaBombard, and J. Terry, "Intermittent fluctuations in the alcator C-mod scrape-off layer for ohmic and high confinement mode plasmas," Physics of Plasmas 25, 056103 (2018).

${ }^{18}$ B. Nold, G. D. Conway, T. Happel, H. W. Müller, M. Ramisch, V. Rohde, , and U. Stroth, "Generation of blobs and holes in the edge of the ASDEX Upgrade tokamak," Plasma Physics and Controlled Fusion 52, 065005 (2010).

${ }^{19}$ S. Zweben, W. Davis, S. Kaye, J. Myra, R. Bell, B. LeBlanc, R. Maqueda, T. Munsat, S. Sabbagh, Y. Sechrest, et al., "Edge and SOL turbulence and blob variations over a large database in NSTX," Nuclear Fusion 55, 093035 (2015).

${ }^{20}$ R. Perkins, J.-W. Ahn, R. Bell, A. Diallo, S. Gerhardt, T. Gray, D. Green, E. Jaeger, J. Hosea, M. Jaworski, et al., "Fast-wave power flow along SOL field lines in NSTX and the associated power deposition profile across the SOL in front of the antenna," Nuclear Fusion 53, 083025 (2013).

${ }^{21}$ J. R. Myra and H. Kohno, "Radio frequency wave interactions with a plasma sheath: The role of wave and plasma sheath impedances," Physics of Plasmas 26, 052503 (2019).

${ }^{22}$ W. Tierens, G. Urbanczyk, L. Colas, , and M. Usoltceva, "Numerical solutions of maxwell's equations in 3D in frequency domain with linear sheath boundary conditions," Physics of Plasmas 26, 083501 (2019).

${ }^{23}$ D. Swain, J. Wilson, P. Ryan, R. Pinsker, M. Carter, D. Gates, G. Hanson, J. Hosea, R. Maqueda, T. Mau, et al., "Results of high-harmonic fast wave experiments on NSTX," in Proc. 27th EPS Conf on Controlled Fusion and Plasma Physics, Budapest (2000).

${ }^{24}$ S. Zweben, R. Maqueda, D. Stotler, A. Keesee, J. Boedo, C. Bush, S. Kaye, B. LeBlanc, J. Lowrance, V. Mastrocola, et al., "High-speed imaging of edge turbulence in NSTX," Nuclear Fusion 44, 134 (2003).

${ }^{25}$ R. Maqueda, G. Wurden, S. Zweben, L. Roquemore, H. Kugel, D. Johnson, S. Kaye, S. Sabbagh, and R. Maingi, "Edge turbulence measurements in NSTX by gas puff imaging," Review of Scientific Instruments 72, 931-934 (2001).

${ }^{26}$ R. Perkins, J. Hosea, G. Kramer, J.-W. Ahn, R. Bell, A. Diallo, S. Gerhardt, T. Gray, D. L. Green, E. F. Jaeger, et al., "High-harmonic fast-wave power flow along magnetic field lines in the scrape-off layer of NSTX," Physical review letters 109, 045001 (2012).

${ }^{27}$ N. Bertelli, E. Jaeger, J. Hosea, C. Phillips, L. Berry, P. Bonoli, S. Gerhardt, D. Green, B. LeBlanc, R. Perkins, et al., "Full wave simulations of fast wave efficiency and power losses in the scrape-off layer of tokamak plasmas in mid/high harmonic and minority heating regimes," Nuclear Fusion 56, 016019 (2015).

${ }^{28}$ R. J. Perkins, J. C. Hosea, G. Taylor, N. Bertelli, G. J. Kramer, Z. P. Luo, C. M. Qin, L. Wang, J. C. Xu, and X. J. Zhang, "Resolving interactions between ion-cyclotron range of frequencies heating and the scrape-off layer plasma in EAST using divertor probes," Plasma Physics and Controlled Fusion 61, 045011 (2019).

${ }^{29}$ P. Tamain, C. Colin, L. Colas, C. Baudoin, G. Ciraolo, R. Futtersack, D. Galassi, P. Ghendrih, N. Nace, F. Schwander, and E. Serre, "Numerical analysis of the impact of an RF sheath on the Scrape-Off Layer in 2D and 3D turbulence simulations," Nuclear Materials and Energy 12, 1171 1177 (2017), proceedings of the 22nd International Conference on Plasma Surface Interactions 2016, 22nd PSI.

${ }^{30}$ L. Lu, L. Colas, J. Jacquot, B. Després, S. Heuraux, E. Faudot, D. V. Eester, K. Crombé, A. Křivská, J.-M. Noterdaeme, W. Helou, and J. Hillairet, "Modelling of radio frequency sheath and fast wave coupling on the realistic ion cyclotron resonant antenna surroundings and the outer wall," Plasma Physics and Controlled Fusion 60, 035003 (2018). 